\title{
ESPECIFICAÇÃO MORFOLÓGICA \\ DE PRONOMES NOMINATIVOS, \\ CONCORDÂNCIA VERBAL \\ E SUJEITOS NULOS EM \\ PORTUGUÊS BRASILEIRO
}

\author{
ESPECIFICACIÓN MORFOLÓGICA DE LOS PRONOMBRES NOMINATIVOS, \\ CONCORDANCIA VERBAL Y SUJETOS NULOS EN PORTUGUÉS BRASILEÑO
}

\author{
MORPHOLOGICAL SPECIFICATION OF NOMINATIVE PRONOUNS, VERBAL AGREEMENT, \\ AND NULL SUBJECTS IN BRAZILIAN PORTUGUESE
}

Jairo Nunes*

Universidade de São Paulo

\begin{abstract}
RESUMO: $\mathrm{O}$ fato de o português brasileiro $(P B)$ não ser uma língua pro-drop canônica é frequentemente associado ao enfraquecimento de seu sistema de concordância verbal. Entretanto, à parte a concordância associada ao pronome $t u$, PB padrão e português europeu padrão exibem o mesmo paradigma de concordância verbal. Neste artigo, argumento que essa discrepância encontra explicação nas especificações dos morfemas de concordância do PB. Mais especificamente, defendo que seus pronomes nominativos são maximamente subespecificados (NUNES, 2019) e isso se reflete nos traços- $\phi$ que T pode portar (FERREIRA, 2000; NUNES, 2008) e em sua valoração via concordância. Assumindo que sujeitos nulos pronominais resultam de uma operação de elipse licenciada quando $T$ tem seu traço- $\phi$ mais proeminente valorado (MARTINS; NUNES, a sair), explica-se a gradação da aceitabilidade de sujeitos pronominais nulos em PB a depender do pronome elidido, bem como a correlação inversa entre possibilidade de sujeito nulo e possibilidade de hiperalçamento (NUNES, 2015).
\end{abstract}

PALAVRAS-CHAVE: Sujeito nulo. Concordância verbal. Português Brasileiro. Hiperalçalmento.

RESUMEN: El hecho de que el portugués brasileño $(\mathrm{PB})$ no es una lengua pro-drop canónica es comúnmente relacionado al empobrecimiento de su sistema de concordancia verbal. Sin embargo, dejando de lado la concordancia asociada al pronombre $t u$, el PB estándar y el portugués europeo estándar muestran el mismo paradigma de concordancia verbal. En este artículo, propongo que esa discrepancia encuentra su explicación en las especificaciones de los morfemas de concordancia de PB. Más específicamente, sostengo que sus pronombres nominativos son máximamente subespecificados (NUNES, 2019) y que esto se refleja en los rasgos$\phi$ que T puede tener (FERREIRA, 2000; NUNES, 2008) y en su valoración vía concordancia. Asumiendo que los sujetos nulos pronominales son el resultado de una operación de elipsis permitida cuando T tiene su rasgo- $\phi$ más saliente valorado (MARTINS;

*Professor do Departamento de Linguística da Faculdade de Filosofia, Letras e Ciências Humanas da Universidade de SãoPaulo.E-mail: jmnunes@usp.br. 
NUNES, a salir), puede explicarse la gradación de aceptabilidad de sujetos pronominales nulos en PB dependiendo del pronombre elidido, así como la correlación inversa entre la posibilidad de sujeto nulo y la posibilidad de hiperelevación (NUNES, 2015).

PALABRAS CLAVE: Sujeto Nulo. Concordancia Verbal. Portugués Brasileño. Hiperelevación.

ABSTRACT: The fact that Brazilian Portuguese (BP) is not a canonical pro-drop language has been often associated with the weakening of its verbal agreement system. However, leaving aside the agreement triggered by the pronoun $t u$, Standard BP and Standard European Portuguese display the same paradigm of verbal agreement inflection. In this paper I argue that this inconsistency may find an explanation in the morphological specifications of agreement morphemes in BP. More specifically, I contend that its nominative pronouns are maximally underspecified (NUNES, 2019), which is reflected on the $\phi$ features that T may bear (FERREIRA, 2000; NUNES, 2008) and how these features get valued under Agree. Assuming that pronominal null subjects result from an ellipsis operation licensed when T has its most prominent $\varnothing$-feature valued (MARTINS; NUNES, forthcoming), I provide an account of the different degrees of acceptability of null subjects in BP depending on the elided pronoun, as well as the inverse correlation between availability of null subjects and availability of hyper-raising.

KEYWORDS: Null Subjects. Verbal Agreement. Brazilian Potuguese. Hyper-raising.

\section{INTRODUÇÃO ${ }^{1}$}

As diferenças entre português brasileiro $(P B)$ e português europeu $(P E)$ no que diz respeito aos seus sujeitos nulos têm sido objeto de uma farta literatura dentro da Teoria de Princípios e Parâmetros ${ }^{2}$. Uma intuição que tem alimentado boa parte dessas pesquisas é que PB deixou de ser uma língua de sujeito nulo canônica em função do enfraquecimento de sua concordância verbal. Esse enfraquecimento é claramente observado em sentenças de PB não-padrão como (1), com "concordância de terceira pessoa do singular" associada a pronomes que desencadeiam outras formas de concordância em PB padrão.

$$
\text { \%PB não-padrão: }
$$

\{Nós/vocês/eles/elas\} dança muito bem.

O problema que essa abordagem enfrenta, no entanto, é que $\mathrm{PB}$ padrão e PE padrão são praticamente idênticos em relação à concordância verbal, como ilustrado na Tabela 1 com o presente do indicativo, mas diferem consideravelmente em relação a seus sujeitos nulos, como se vê em (2).

\begin{tabular}{c|c}
$\begin{array}{c}\text { Pronomes } \\
\text { nominativos }\end{array}$ & $\begin{array}{c}\text { Presente do } \\
\text { Indicativo }\end{array}$ \\
\hline$e u$ & danço \\
\hline$t u(\mathrm{PE})$ & danças \\
\hline você & dança
\end{tabular}

\footnotetext{
${ }^{1}$ Este trabalho faz um apanhado de resultados de trabalhos recentes que tenho desenvolvido individualmente e em parceria com Ana Maria Martins e Mary A. Kato, a quem muito agradeço. Muito obrigado também a Janayna Carvalho e Ezekiel Panitz por seus comentários à primeira versão deste texto. Finalmente, gostaria de agradecer o apoio recebido do $\mathrm{CNPq}$ (processo 303195/2019-3).

${ }^{2}$ Vejam-se, entre outros, Chao (1983); Moreira da Silva (1983); Negrão (1986); Galves (1987, 1993, 1998), Duarte (1993, 1995, 2000), Figueiredo Silva (1996), Kato (1999, 2000, 2002), Ferreira (2000, 2009), Kato e Negrão (2000), Modesto (2000), Barbosa, Duarte e Kato (2001, 2005), Rodrigues (2002, 2004), Holmberg, Nayudu e Sheehan (2009), Petersen (2011), Saab (2016), Nunes (2019), Kato, Martins e Nunes, a sair; e Martins e Nunes, a sair.
} 


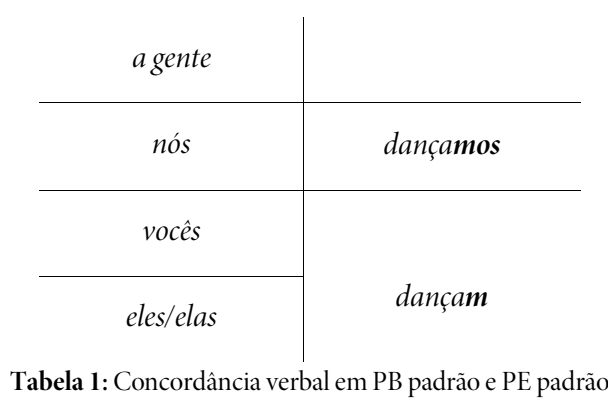

Fonte: produzido pelo autor

(2)
a. [O que $\emptyset$ tenho a ver com isso]?
b. [O que $\emptyset_{\text {viste]? }}$
c. [O que $\emptyset$ quer fazer]?
d. [O que $\varnothing$ fez desta vez]?
e. [Quem $\emptyset$ devíamos contratar]?
f. [Quando Ø deve viajar]?
g. [Quando Ø viajaram]?
h. [Quem $\emptyset$ contrataram]?

$(\varnothing=e u \rightarrow$ PE: OK; PB: ??)

$(\varnothing=t u \rightarrow \mathrm{PE}: \mathrm{OK})$

$\left(\varnothing=\right.$ voce $\rightarrow$ PE: $\left.\mathrm{OK} ; \mathrm{PB}:{ }^{\star}\right)$

$\left(\varnothing=\right.$ ele $/$ ela $\rightarrow$ PE: OK; PB: $\left.{ }^{*}\right)$

$(\varnothing=$ nós $\rightarrow$ PE: $\mathrm{OK} ; \mathrm{PB}: \mathrm{OK})$

$\left(\varnothing=\right.$ a gente $\left.\rightarrow \mathrm{PE}{ }^{\star} ; \mathrm{PB}:{ }^{\star}\right)$

$(\varnothing=$ vocês $\rightarrow$ PE: $\mathrm{OK} ; \mathrm{PB}:$ ?? $)$

$(\varnothing=$ eles/elas $\rightarrow$ PE: OK; PB: ??)

A única diferença entre PB padrão e PE padrão na Tabela 1 diz respeito ao pronome tu e sua correspondente concordância verbal. Como é de conhecimento geral, a forma nominativa $t u$ desapareceu na maioria dos dialetos do PB e nos dialetos em que é produtivamente empregado, a concordância de segunda pessoa do singular cede espaço à concordância de "terceira pessoa do singular", como em $(1)^{3}$. Ressalte-se que as sentenças em (2) envolvem um inequívoco ambiente de sujeito nulo pronominal, pois o constituinte interrogativo bloqueia a possibilidade de o sujeito nulo ser analisado como um caso de topic drop ${ }^{4}$. Surge então a pergunta de por que PB padrão e PE padrão têm comportamento distinto em relação ao licenciamento de sujeitos nulos, mas comportamento idêntico em relação à concordância verbal.

A pergunta se torna ainda mais instigante quando examinamos alguns detalhes do paradigma em (2). Observando-se PE isoladamente, nota-se um comportamento bastante uniforme: com exceção do pronome a gente (veja-se (2f)), todos os demais pronomes podem ter suas formas nulas licenciadas. Já PB exibe um maior leque de graus de aceitabilidade, indo do totalmente aceitável (a contraparte nula de nós) ao totalmente inaceitável (as contrapartes nulas de você, ele/ela e a gente), passando pela marginalidade das contrapartes nulas de eu, vocês e eles/elas). O paradigma é também surpreendente de um ponto de vista funcional. Em PB, os morfemas -o e -mos são os únicos que identificam inequivocamente o sujeito; entretanto, -o só licencia marginalmente a contraparte nula de $e u$, comportando-se de modo mais similar ao morfema - $m$, que pode licenciar (marginalmente) mais de um sujeito nulo. Por outro lado, o morfema- $\varnothing$ em PE licencia a contraparte nula de você e ele/ela, mas não a contraparte de a gente.

Há também uma interessante correlação em PB entre licenciamento de um sujeito nulo pronominal e hiperalçamento ${ }^{5}$ : de um modo geral, um pronome pode sofrer hiperalçamento se a sua contraparte nula não for independentemente licenciada e vice-versa. Assim, os pronomes você(s), a gente, ele(s) e ela(s) do PB, cujas contrapartes nulas em (2) são inaceitáveis ou marginais, podem sofrer hiperalçamento, como ilustrado em (3a) e (3b) abaixo. Por sua vez, o hiperalçamento de nós, que pode ter sua contraparte nula licenciada (veja-se (2e)), gera o maior grau de inaceitabilidade (veja-se (3d)). Finalmente, o pronome eu, cuja contraparte nula é

\footnotetext{
${ }^{3}$ Vejam-se, por exemplo, Menon e Lorengian-Penkal (2002) e Paredes Silva (2003).

${ }^{4}$ Vejam-se Modesto (2000), Ferreira (2000), Rodrigues (2004) e Nunes (2008) para discussão relevante.

${ }^{5}$ O termo hiperalçamento (ver URA, 1994) tem sido usado para descrever movimento-A a partir de um domínio finito ou com infinitivo flexionado. Para discussão relevante sobre PB, vejam-se entre outros, Ferreira (2000, 2009), Duarte (2004), Martins e Nunes (2005, 2010) e Nunes (2008, 2015, 2019).
} 
marginal (veja-se (2a)), não recebe um tratamento uniforme entre os falantes no que diz respeito a hiperalçamento (veja-se (3c)). Para alguns falantes (entre os quais eu me incluo), (3c) é tão inaceitável quanto (3d); para outros, a aceitabilidade de (3c) se aproxima de $(3 a)$ e $(3 b)^{6}$.

PB:

a. $\{$ Você/a gente/ele/ela\} parece que pegou o caminho errado.

b. \{Vocês/eles/elas\} parecem que pegaram o caminho errado.

c. \%Eu pareço que peguei o caminho errado.

d. ${ }^{\star}$ Nós parecemos que pegamos o caminho errado.

Neste artigo buscarei resgatar a intuição de que o licenciamento marginal de sujeitos nulos em PB está associado ao enfraquecimento de sua concordância, analisando esse "enfraquecimento" de modo que também possa se aplicar ao PB padrão e capturar a correlação entre os padrões de aceitabilidade vistos em (2) e (3). O artigo está organizado da seguinte forma: na seção 2, mostro como o paradigma em (2) pode ser derivado em PE e PB se assumirmos a análise de sujeitos nulos pronominais em termos de elipse advogada por Martins e Nunes (a sair) e a proposta de Nunes (2019) de que o sistema pronominal do PB passou por um processo geral de subespecificação em relação aos traços de número e pessoa; na seção 3, mostro como o padrão de hiperalçamento ilustrado em (3) se segue da proposta de Ferreira $(2000,2009)$ de que T em PB pode estar associado a um conjunto completo ou incompleto de traços- $\phi$, se implementada como em Nunes (2008); a seção 4 apresenta evidência independente para os diferentes ingredientes utilizados nas seções precedentes e a seção 5 conclui o trabalho.

\section{SUBESPECIFICACÃO DOS PRONOMES NOMINATIVOS EM PB E PRO-DROP COMO ELIPSE}

Consideremos primeiramente os traços de número e pessoa dos pronomes nominativos da Tabela 1 em PE. Podemos identificar dois grupos de um ponto de vista morfológico. O primeiro grupo envolve os pronomes você, ele e ela, que têm sua forma de plural derivada da adição regular do morfema de plural -s (vocês, eles e elas). O segundo grupo, formado por eu, tu, nós e a gente, não tem sua informação de número independente da informação de pessoa. Em outras palavras, os traços de pessoa e número são independentes no primeiro grupo (P-N), mas se apresentam fundidos no segundo grupo (P.N). Examinemos agora o valor desses traços no segundo grupo. Para distinguir morfologicamente $e u$, tu e nós, faz-se necessário lançar mão de dois valores para pessoa e dois valores para número: assim, os valores 1 e 2 para pessoa distinguem eu de tu e os valores SG e PL distinguem eu de nós (eu: 1.SG; tu: 2.SG: nós: 1.PL). Já o pronome a gente pode ser morfologicamente identificado se seus traços fundidos de pessoa e número forem desprovidos de valor (a gente: P.N), o que se coaduna com o fato de não haver nenhum morfema de concordância específico para $a$ gente. Voltando aos demais pronomes do primeiro grupo, os valores 2 e 3 distinguem respectivamente os pronomes você/vocês de ele/eles/elas/elas; os valores SG e PL para número distinguem você/ele/ela de vocês/eles/elas; e finalmente os valores MASC e FEM para gênero distinguem os pronomes ele/eles de ela/elas. Essas especificações, retratadas na Tabela 2, estão associadas às regras de correspondência morfológica para a flexão de concordância verbal em (4), derivando o padrão de concordância ilustrado na Tabela $1^{7}$.

\footnotetext{
${ }^{6}$ Neste artigo deixarei de lado sentenças como (i), que Martins e Nunes (2010) analisam como envolvendo hiperalçamento de um tópico gerado na oração encaixada. Observe-se que sentenças como (i) são aceitáveis mesmo para falantes que não permitem (3c). Para discussão das propriedades de sentenças como (i), vejam-se Martins e Nunes $(2005,2010)$ e Nunes $(2008,2016)$
}

(i) Eui pareço que $\left[t_{\mathrm{i}}\right.$ [eu peguei o caminho errado]]

${ }^{7}$ Para detalhes e discussão relevante, veja-se Kato, Martins e Nunes, a sair. Na seção 4 abaixo, discutirei como se dá a concordância com eu em tempos verbais que não dispõem de morfema de concordância específico para esse pronome. 


\begin{tabular}{|c|c|}
\hline $\begin{array}{c}\text { Pronomes } \\
\text { nominativos }\end{array}$ & $\begin{array}{l}\text { Especificação } \\
\text { Morfológica }\end{array}$ \\
\hline$e u$ & P.N:1.SG \\
\hline$t u$ & P.N:2.SG \\
\hline você & $\mathrm{P}: 2$ - N:SG \\
\hline ele & P:3 - G:MASC - N:SG \\
\hline ela & P:3 - G:FEM - N:SG \\
\hline agente & P.N \\
\hline nós & P.N:1.PL \\
\hline vocês & $\mathrm{P}: 2$ - N:PL \\
\hline eles & P:3 - G:MASC - N:PL \\
\hline elas & P:3 - G:FEM - N:PL \\
\hline
\end{tabular}

Tabela 2: Especificação morfológica dos pronomes nominativos em PE Fonte: produzida pelo autor

(4) Regras de realização morfológica para a flexão de concordância verbal em PE:

a. $\quad[P . N: 1 . P L] \leftrightarrow\{-\mathrm{mos}\}$

b. $\quad[\mathrm{N}: \mathrm{PL}] \leftrightarrow\{-\mathrm{m}\}$

c. $[$ P.N:1.SG $] \rightarrow\{-0\} /$ PRES.INDIC

$\rightarrow\{-\mathrm{i}\} \quad$ nos demais contextos.

d. $[$ P.N:2.SG $] \rightarrow\{-$ ste $\} /$ PRET.PERF.INDIC

$\rightarrow\{-s\} \quad$ nos demais contextos.

e. Ø nos demais contextos.

Nunes (2019) propõe que houve um maciço processo de subespecificação morfológica no sistema pronominal do PB, eliminando especificações redundantes. Com a perda de $t u$, não se faz mais necessário lançar mão de dois valores para o traço de pessoa para distinguir individualmente os pronomes com traços de pessoa e número fundidos: o valor SG é suficiente para identificar eu (P.N:SG), já que o traço de número de a gente é desprovido de valor (P.N); sendo assim, nós pode ser minimamente especificado só com um valor para o traço de pessoa (P.N:1). Explorando essa hipótese de máxima subespecificação em PB, Nunes (2019) argumenta que os demais pronomes podem também ser devidamente identificados morfologicamente mesmo sem valor para o traço de pessoa ou valor de SG para número, como esquematizado na Tabela 3. Consoantemente, as regras de realização morfológica para a flexão de concordância verbal em PB foram também reanalisadas como em (5) (veja-se nota 7). 


\begin{tabular}{c|c}
$\begin{array}{c}\text { Pronomes } \\
\text { nominativos }\end{array}$ & $\begin{array}{c}\text { Especificação } \\
\text { Morfológica }\end{array}$ \\
\hline$e u$ & P.N:SG \\
\hline você & P - N \\
\hline ele & P - G:MASC - N \\
\hline ela & P - G:FEM - N \\
\hline agente & P.N \\
\hline nós & P.N:1 \\
\hline vocês & P - N:PL \\
\hline eles & P - G:MASC - N:PL \\
\hline elas & P - G:FEM - N:PL \\
\hline
\end{tabular}

Tabela 3: Especificação morfológica dos pronomes nominativos em PB Fonte: produzida pelo autor

(5) Regras de realização morfológica para a flexão de concordância verbal em PB:
a. $[\mathrm{P} / \mathrm{N}: 1] \leftrightarrow\{-\mathrm{mos}\}$
b. $[\mathrm{N}: \mathrm{PL}] \leftrightarrow\{-\mathrm{m}\}$
c. $[\mathrm{P} / \mathrm{N}: \mathrm{SG}] \rightarrow\{-\mathrm{o}\} / \mathrm{PRES}$.INDIC
$\rightarrow\{-i\} /$ nos demais contextos.

d. $\varnothing$ nos demais contextos.

O que é crucial para a presente discussão é que as regras de correspondência morfológica em (4) e (5) produzem as mesmas formas de concordância quando respectivamente aplicadas às Tabelas 2 e 3 , como ilustrado na Tabela 4 com o presente do indicativo, apesar de os pronomes terem especificações morfológicas distintas em cada dialeto. 


\begin{tabular}{|c|c|c|c|c|c|}
\hline \multirow[b]{2}{*}{$\begin{array}{c}\text { pronomes } \\
\text { nominativos }\end{array}$} & \multicolumn{2}{|l|}{ PE } & \multicolumn{2}{|l|}{ PB } & \multirow[b]{2}{*}{$\begin{array}{l}\text { PRES. } \\
\text { INDIC. }\end{array}$} \\
\hline & especificação morfológica & $\begin{array}{l}\text { valoração dos } \\
\text { traços- } \phi \text { de T }\end{array}$ & $\begin{array}{l}\text { especificação } \\
\text { morfológica }\end{array}$ & $\begin{array}{l}\text { valoração dos } \\
\text { traços- } \phi \text { de T }\end{array}$ & \\
\hline$t u$ & P.N:2.SG & P.N:2.SG & & & danças \\
\hline nós & P.N:1.PL & P.N:1.PL & P.N:1 & P.N:1 & dançamos \\
\hline$e u$ & P.N:1.SG & P.N:1.SG & P.N:SG & P.N:SG & danço \\
\hline você & P:2 - N:SG & $P: 2$ - N:SG & $\mathrm{P}-\mathrm{N}$ & \multirow{4}{*}{ P:u - N:u } & \multirow{4}{*}{ dança } \\
\hline ele & P:3 - G:MASC - N:SG & \multirow{2}{*}{$\mathrm{P}: 3$ - N:SG } & P - G:MASC - N & & \\
\hline ela & P:3 - G:FEM - N:SG & & P - G:FEM - N & & \\
\hline agente & P.N & $\mathrm{P}: \mathrm{u}-\mathrm{N}: \mathrm{u}$ & P.N & & \\
\hline vocês & $\mathrm{P}: 2$ - N:PL & $\mathrm{P}: 2$ - N:PL & $P$ - N:PL & \multirow{3}{*}{ P:u - N:PL } & \multirow{3}{*}{ dançam } \\
\hline eles & P:3 - G:MASC - N:PL & \multirow{2}{*}{$\mathrm{P}: 3$ - N:PL } & P - G:MASC - N:PL & & \\
\hline elas & P:3 - G:FEM - N:PL & & P - G:FEM - N:PL & & \\
\hline
\end{tabular}

Tabela 4: Especificações morfológicas de pronomes nominativos e valoração de T em PE e PB

Fonte: produzida pelo autor

Essa abordagem captura, portanto, a intuição de que há um "enfraquecimento" na concordância verbal de PB, mesmo na modalidade padrão. Mais importante que ter distintos expoentes fonológicos para a flexão de concordância é a especificação dos traços subjacentes à realização fonológica. Especificamente, Martins e Nunes (a sair) argumentam que sujeitos nulos pronominais são resultado de uma operação de elipse, ${ }^{8}$ restringida pela condição em (6), em que proeminência é especificada como em (7):

(6) Condição de Valoração do Traço Proeminente:

T pode licenciar a elipse de um sujeito pronominal PRON se:

(i) T estiver associado a um traço-E (veja-se MERCHANT, 2001); e

(ii) concordância entre T e PRON envolver valoração do traço mais proeminente de T.

Proeminência: pessoa $>$ número $>$ gênero $>$ Caso

Relevante para a presente discussão são os traços de pessoa e número, sendo o primeiro mais proeminente que o segundo. Como se pode ver na terceira coluna da Tabela 4, T em PE tem sempre seu traço de pessoa valorado, exceto quando o sujeito é o pronome $a$ gente; assim, só o pronome a gente não pode ser elidido (veja-se (2f)). Em PB, por outro lado, T só tem seu traço de pessoa valorado quando o sujeito é o pronome nós; portanto, só esse pronome pode ser elidido e produzir um resultado plenamente aceitável (vejase (2e)). Os casos em que o traço de número de T (o segundo mais proeminente na hierarquia de (7)) é valorado, como ocorre com os pronomes eu, vocês, eles e elas, produzem um resultado de aceitabilidade marginal (vejam-se (2a), (2g) e (2h)). Por fim, os casos de sujeito nulo com os pronomes você, a gente, ele e ela, em que nenhum traço de T é valorado, produzem resultados inaceitáveis (vejam-se (2c), (2d) e (2f)). 
A análise acima captura, portanto, o fato de PB padrão e PE padrão terem basicamente o mesmo paradigma de concordância verbal, mas terem comportamento semelhante no que diz respeito ao licenciamento de sujeitos nulos somente no caso dos pronomes nós e a gente.

\section{SUBESPECIFICAÇÃO DOS PRONOMES NOMINATIVOS EM PB E HIPERALÇAMENTO}

Consideremos agora a correlação entre (2) e (3) em PB. Tendo-se em conta a restrita aceitabilidade de sujeito nulos pronominais em PB vista em (2), surge então a questão de qual é a natureza do sujeito nulo de uma sentença como (8) abaixo, por exemplo, em PB.

PB:

[A Joana $]_{\mathrm{w}}$ disse que [a irmã d[a Maria $\left.]_{\mathrm{k}}\right]_{\mathrm{i}}$ acha que $\emptyset_{\mathrm{i} /{ }^{\star} \mathrm{k} /{ }^{/ \mathrm{w}} /{ }^{*} \mathrm{y}}$ está grávida.

O sujeito nulo de (8) tem de necessariamente tomar como antecedente o constituinte c-comandante mais próximo. Assim, embora haja três possíveis antecedentes pragmaticamente compatíveis com o predicado grávida em (8), o sujeito nulo da subordinada só pode ser interpretado como se referindo à irmã da Maria.

Pondo de lado o fato de que a oração subordinada de (8) é finita, a interpretação de seu sujeito nulo se assemelha à interpretação observada em estruturas envolvendo casos típicos de controle obrigatório. Como base nesse tipo de similaridade, Kato (1999) propõe que o sujeito nulo de sentenças como (8) é de fato um PRO e, portanto, deve ter sua interpretação estabelecida pelo módulo de controle da gramática. Assumindo a Teoria de Controle por Movimento de Hornstein (1999, 2001), Ferreira (2000, 2009) e Rodrigues $(2002,2004)$ reinterpretam a análise de Kato em termos de movimento. Ou seja, o sujeito nulo de sentenças como (8) é analisado como um vestígio de movimento-A (uma cópia apagada) e, enquanto tal, deve encontrar seu antecedente numa posição de c-comando local.

Essa reinterpretação, por sua vez, requer uma reanálise das orações finitas em PB. Dentro do modelo de Chomsky (2001), por exemplo, a Condição de Ativação bloqueia movimento-A a partir de uma posição marcada com Caso e Caso é interpretado como reflexo de concordância com uma sonda portando um conjunto "completo" de traços- $\phi$. Dentro dessa abordagem, o Caso nominativo, por exemplo, é reflexo da concordância entre os traços- $\phi$ do sujeito e os traços- $\phi$ de T (tipicamente, pessoa e número). Sob essa perspectiva, se o sujeito nulo em (8) é um vestígio de movimento-A, a posição de sujeito da oração subordinada não pode ter sido marcada com Caso. Essa conclusão leva Ferreira $(2000,2009)$ a postular que T finito em PB pode estar associado a um conjunto completo ou a um conjunto incompleto de traços- $\phi$. Nesta última situação, o sujeito não tem seu Caso valorado e pode sofrer movimento-A. Nunes (2008) desenvolve a proposta de Ferreira, argumentando que T finito em PB pode portar traços de pessoa e número ou só de número. Se T porta ambos os traços, vai valorar o Caso de seu sujeito, congelando-o para efeito de movimento-A; por outro lado, se T só dispõe de traço de número, seu sujeito não vai ter seu Caso valorado e poderá, portanto, sofrer movimento-A. Nessa análise, o núcleo T da oração subordinada de (8) porta somente um traço de número, permitindo que seu sujeito se mova para a oração matriz, onde pode ter seu traço de caso valorado se T portar traços de pessoa e número. Crucialmente, com exceção da flexão de concordância desencadeada por nós, todas as demais flexões podem ser descritas em termos de número e pessoa ou só de número, como ilustrado na Tabela 5. 


\begin{tabular}{|c|c|c|c|c|}
\hline $\begin{array}{c}\text { Pronomes } \\
\text { nominativos }\end{array}$ & $\begin{array}{l}\text { Especificação } \\
\text { morfológica }\end{array}$ & $\begin{array}{c}\text { Valoração de T } \\
\text { completo } \\
\text { (P:u - N:u) }\end{array}$ & $\begin{array}{c}\text { Valoração de T } \\
\text { incompleto } \\
\text { (N:u) }\end{array}$ & $\begin{array}{l}\text { PRES. } \\
\text { INDIC. }\end{array}$ \\
\hline você & $\mathrm{P}-\mathrm{N}$ & \multirow{4}{*}{$\mathrm{P}: \mathrm{u}-\mathrm{N}: \mathrm{u}$} & \multirow{4}{*}{$\mathrm{N}: \mathrm{u}$} & \multirow{4}{*}{ dança } \\
\hline ele & P - G:MASC - N & & & \\
\hline ela & P - G:FEM - N & & & \\
\hline agente & P.N & & & \\
\hline vocês & $P-N: P L$ & \multirow{3}{*}{$P: u-N: P L$} & \multirow{3}{*}{$\mathrm{N}: P L$} & \multirow{3}{*}{ dançam } \\
\hline eles & P - G:MASC - N:PL & & & \\
\hline elas & P - G:FEM - N:PL & & & \\
\hline nós & P.N:1 & P.N:1 & * & dançamos \\
\hline \multirow{2}{*}{$e u$} & \multirow{2}{*}{ P.N:SG } & \multirow{2}{*}{ P.N:SG } & $\%{ }^{*}$ & \multirow{2}{*}{ danço } \\
\hline & & & $\mathrm{N}: S G$ & \\
\hline \multicolumn{5}{|c|}{ Tabela 5: Ambiguidade de T finito em PB } \\
\hline
\end{tabular}

De acordo com a Tabela 5, se T entra na numeração com pessoa e número, esses traços vão ser valorados como esquematizado na terceira coluna a depender da especificação morfológica do sujeito ${ }^{9}$. As regras de correspondência morfológica em (5) produzem, então, as formas da última coluna, exemplificada com o presente do indicativo. Nessa situação, os sujeitos têm seu traço de Caso valorado como nominativo e se tornam indisponíveis para efeito de movimento-A. Relevante para os nossos propósitos é a situação em que T só dispõe de traço de número, como retratado na quarta coluna. Nessa circunstância, há três resultados possíveis, como veremos em detalhe abaixo.

No caso dos pronomes você, a gente, ele, e ela, seus traços de número não têm valor em PB e, portanto, não podem valorar o traço de número de T. Só há, portanto, uma checagem por compatibilidade (matching) entre os traços do sujeito e T e o traço de número de T permanece sem valoração. A regra de correspondência morfológica em (5d) é aplicada, nenhum expoente fonológico é atribuído à especificação N:u e o verbo se superficializa sem nenhuma marca de concordância (dança). Como o sujeito não teve seu traço de Caso valorado, pode então se mover para a oração subordinante (se houver) e ter seu Caso valorado por T se T dispuser de um conjunto completo de traços- $\phi$. Em outras palavras, a previsão é que hiperalçamento com esses pronomes deve produzir resultados aceitáveis. Como vimos em (3a), repetido aqui em (9), a previsão é confirmada. $\mathrm{PB}$ :

$\{\text { Você/a gente/ele/ela }\}_{\mathrm{i}}$ parece que $t_{\mathrm{i}}$ pegou o caminho errado.

Situação semelhante é a dos pronomes vocês, eles e elas. Se forem sujeitos de um T portador de número somente, esse traço vai ser valorado como PL e a regra de correspondência em (5b) vai fazer com que o verbo dançar na Tabela 5 se superficialize como dançam. Como não têm seu traço de Caso valorado nesse cenário, esses pronomes podem também se mover para a oração subordinante em busca de valor para seu traço de Caso. Assim, esses pronomes também podem ser alvo de hiperalçamento, como visto em (3b), repetido aqui como (10).

${ }^{9}$ A concordância entre T completo e os pronomes eu e nós na Tabela 5 requer que os traços de pessoa e número em T sofram fusão. Observe-se que essa fusão está tacitamente assumida em qualquer análise desse tipo de concordância em PE. 
PB:

$\{\text { Vocês/eles/elas }\}_{\mathrm{i}}$ parecem que $t_{\mathrm{i}}$ pegaram o caminho errado.

O segundo padrão envolve o pronome nós. Evidentemente, se nós for o sujeito de um T com pessoa e número, o pronome vai ter seu traço de Caso valorado e se tornar indisponível para movimento-A. O que falta determinar é o que ocorre se T só possui traço de número. Presumivelmente, tem-se aí uma situação de incompatibilidade (mismatch) de traços, pois o traço de número de nós se encontra fundido ao traço de pessoa. Além disso, de acordo com Nunes (2019), nós em PB apresenta 1 como valor para o conglomerado P.N e esse não pode ser um valor para número em T. Posto em outras palavras, isso significa que não há como se obter o morfema -mos a partir da regra de correspondência em (5a) se T dispuser apenas do traço de número. Portanto, nenhuma derivação convergente pode resultar na sentença (3d), repetida abaixo em (11). Se T encaixado tiver número e pessoa, nós tem seu Caso valorado na oração subordinada e hiperalçamento viola a Condição de Ativação de Chomsky (2001); por outro lado, se T encaixado só tiver traço de número, permitindo portanto hiperalçamento de nós, há uma incompatibilidade entre os traços de nós e de T e não se satisfazem as condições para que a regra de correspondência em (5a) se aplique e a forma pegamos seja derivada.

PB:

${ }^{\star}$ Nós parecemos que $t_{\mathrm{i}}$ pegamos o caminho errado.

Consideremos, finalmente, o pronome eu enquanto sujeito de um T com um traço de número somente. Recorde-se que, de acordo com Nunes (2019), o conglomerado resultante da fusão de pessoa e número para eu em PB tem SG como valor (P.N:SG). Nessa situação, duas possibilidades lógicas devem ser contempladas. Na primeira, haveria uma incompatibilidade (mismatch) entre os traços de T e eu, já que o traço de número em eu se apresenta fundido ao traço de pessoa. Em outras palavras, essa incompatibilidade seria análoga ao caso de nós, discutido acima. A outra possibilidade lógica é que uma vez que o valor dos traços de eu é SG e esse é um valor admissível para um T com traço de número exclusivamente, poderia haver valoração de T. Interessantemente, essas duas possibilidades se refletem na divisão de julgamentos entre os falantes. Falantes que parecem estar mais sintonizados à compatibilização dos atributos não admitem que um traço de número fundido com um traço de pessoa (P.N) seja pareado com um traço de número independente. Para esses falantes, a derivação de uma sentença como (3c), repetida em (12), não converge porque a falta de compatibilização entre os traços de eu e T impede que a regra de correspondência em (5c) se aplique e que a forma peguei seja produzida. Outros falantes parecem estar mais sensíveis ao valor dos atributos e como SG é uma opção para o traço de número de T, a valoração ocorre, derivando a forma peguei; nessa derivação, eu não tem seu traço de Caso valorado na oração encaixada e tem de se mover para a oração subordinante em busca de Caso. Para esses falantes, portanto, hiperalçamento com eu é permitido.

PB:

$\% \mathrm{Eu}_{\mathrm{i}}$ pareço que $t_{\mathrm{i}}$ peguei o caminho errado.

Explica-se, assim, a intrigante relação inversa entre licenciamento de sujeito nulo e hiperalçamento. Para que hiperalçamento seja em princípio possível, T não pode portar traço de pessoa, em consonância com a Condição de Ativação. Por outro lado, de acordo com Martins e Nunes (a sair), elipse pronominal só é possível em sentenças finitas se o traço mais proeminente, a saber, o traço de pessoa, for valorado. Segue-se daí que, em $\mathrm{PB}$, os piores candidatos à elipse pronominal são os melhores candidatos para se submeter a hiperalçamento e vice-versa.

\section{EMPOBRECIMENTO MORFOLÓGICO, ELIPSE PRONOMINAL E HIPERALÇAMENTO}

Evidência independente para a abordagem delineada acima é fornecida por tempos com paradigmas de flexão de concordância empobrecidos. Tanto em PE quanto em PB, os únicos tempos verbais que possuem um morfema específico de concordância para o pronome eu são o presente do indicativo (-o), o pretérito perfeito do indicativo (-i) e o futuro do presente (-i). Em todos os demais tempos, eu se comporta como você, a gente, ele e ela, não estando associado a nenhuma marca aberta de concordância. Em Kato, Martins e Nunes (a sair), propõe-se que essa defectividade se segue de uma operação de empobrecimento (no sentido de BONET, 
1991) que apaga a especificação de número de T nos tempos relevantes, quando T entra numa relação de concordância com $e u .{ }^{10}$ Esse apagamento, seja nas regras de correspondência morfológica em (4) para PE seja em (5) para PB, repetidas abaixo em (13) e (14), faz com que a especificação resultante caia na regra geral (a elsewhere condition em (13e) e (14d)) e a ela não é atribuído nenhum expoente fonológico, como respectivamente esquematizado em (15) e (16).

(13) Regras de realização morfológica para a flexão e concordância verbal em PE:

a. $[\mathrm{P} / \mathrm{N}: 1 . \mathrm{PL}] \leftrightarrow\{-\mathrm{mos}\} ;$

b. $[\mathrm{N}: \mathrm{PL}] \leftrightarrow\{-\mathrm{m}\}$

c. $[$ P.N:1.SG] $\rightarrow\{-0\} /$ PRES.INDIC

$\rightarrow\{-\mathrm{i}\} \quad$ nos demais contextos

d. [P.N:2.SG] $\rightarrow\{$-ste $\} /$ PRET.PERF.INDIC

$\rightarrow\{-\mathrm{s}\}$ nos demais contextos.

e. Ø nos demais contextos.

(14) Regras de realização morfológica para a flexão de concordância verbal em PB:

a. $[\mathrm{P} . \mathrm{N}: 1] \leftrightarrow\{-\mathrm{mos}\}$

b. $[\mathrm{N}: \mathrm{PL}] \leftrightarrow\{-\mathrm{m}\}$

c. $[\mathrm{P} . \mathrm{N}: \mathrm{SG}] \rightarrow\{-\mathrm{O}\} /$ PRES.INDIC

$\rightarrow\{-i\} /$ nos demais contextos.

d. Ø nos demais contextos.

(15) Empobrecimento em PE:
a. $\left[e u\left(\right.\right.$ P.N:1.SG) ... T T:u; N:u ...] $\rightarrow_{\text {Agree }}$
b. $\left[e u(P . N: 1 . S G) \ldots T_{\mathrm{P}: 1 ; \mathrm{N}: S G} \ldots\right] \rightarrow \rightarrow_{\text {Empobrecimento }}$
c. $\left[e u(P . N: 1 . S G) \ldots T_{\text {P:1 } 1 . .]} \rightarrow(13 \mathrm{e})\right.$
d. $[\mathrm{P}: 1] \rightarrow \varnothing$

(16) Empobrecimento em PB:
a. [eu (P.N:SG) ... T T:u; N:u ...] $\rightarrow_{\text {Agree }}$
b. $\left[e u(\mathrm{P} . \mathrm{N}: \mathrm{SG}) \ldots \mathrm{T}_{\mathrm{P}: \mathrm{u} ; \mathrm{N}: \mathrm{SG}} . ..\right] \rightarrow_{\text {Empobrecimento }}$
c. $\left[e u(P . N: S G) \ldots T_{P: u} \ldots\right] \rightarrow_{(14 d)}$
d. $[\mathrm{P}: \mathrm{u}] \rightarrow \varnothing$

Consideremos agora o impacto de (15) e (16) em relação ao licenciamento de sujeitos nulos. No caso de PE em (15), o empobrecimento não tem nenhuma consequência na medida em que o traço de pessoa sobrevivente está valorado; portanto, a previsão é que a contraparte nula de eu possa ser licenciada em PE em observância à Condição de Valoração do Traço Proeminente em (6), mesmo em tempos sujeitos a empobrecimento. Já no caso de PB em (16), a regra de empobrecimento elimina a única especificação valorada de T; portanto, a previsão é que a contraparte nula de $e u$ nesses tempos em $\mathrm{PB}$ não seja licenciada nem marginalmente. Kato, Martins e Nunes (a sair) registram que os dados em (17) mostram que ambas as previsões estão corretas:

$$
\begin{aligned}
& \varnothing=e u \rightarrow P E: ~ \vee ; P B: * \\
& \text { a. Todos achavam [que } \emptyset \text { trabalhava na fábrica] } \\
& \text { b. Eles não imaginam [o que } \emptyset \text { gostaria de fazer] } \\
& \text { c. O diretor quer [que } \emptyset \text { contrate outra secretária] }
\end{aligned}
$$

\footnotetext{
${ }^{10}$ Conforme apontado em Kato, Martins e Nunes, a sair, isso implica que esse regra de empobrecimento deve se aplicar antes da fusão entre os traços de pessoa e número de T (veja-se nota 9).
} 
d. O professor esperava [que $\emptyset$ estudasse mais]

e. [Se Ø não ultrapassar os $120 \mathrm{~km} / \mathrm{h}$ ], a gasolina vai dar.

Voltando à correlação entre licenciamento de sujeito nulo e hiperalçamento em PB, se a contraparte de eu não pode ser licenciada em $\mathrm{PB}$ nos tempos sujeitos a empobrecimento, a previsão é que hiperalçamento com eu deve ser permitido nesses tempos. Essa previsão também se confirma. Nunes (2015) mostra que mesmo falantes que não admitem hiperalçamento de eu em orações com marca de concordância na encaixada permitem hiperalçamento com tempos que sofrem empobrecimento, como ilustrado em (18) pelo contraste entre hiperalçamento a partir de uma oração no presente do indicativo e hiperalçamento a partir de orações com o imperfeito do indicativo ou o infinitivo flexionado:
$\mathrm{PB}$
a. \% $\mathrm{Eu}_{\mathrm{i}}$ pareço que $t_{\mathrm{i}}$ nado mal em competição.
b. Eu $u_{i}$ parecia que $t_{i}$ nadava mal em competição.
c. Eu $\mathrm{i}_{\mathrm{i}}$ sou fácil de $t_{\mathrm{i}}$ nadar mal em competição.

Recorde-se que a inaceitabilidade de (18a) para alguns falantes (veja-se nota 6) resulta da falta de compatibilização entre os traços de pessoa e número fundidos em eu e o traço de número independente em T. Se empobrecimento elimina a especificação de número de T em (18b) e (18c), nenhum problema de incompatibilização aparece e as derivações convergem mesmo para falantes que não admitem (18a).

\section{CONSIDERAÇÕES FINAIS}

A literatura sobre sujeitos nulos em PB tem dedicado maior atenção à distribuição e à interpretação dos sujeitos nulos de terceira pessoa do singular. Neste artigo empreendi uma comparação mais sistemática entre os sujeitos nulos de várias pessoas,

demonstrando que um complexo padrão de graus de aceitabilidade a depender do pronome e do tempo verbal relevantes se segue da interação de quatro fatores independentes: (ii) sujeitos nulos pronominais são resultado de uma operação elipse licenciada por um T portando seu traço- $\phi$ mais proeminente valorado (veja-se MARTINS; NUNES a sair); (ii) pronomes nominativos em PB são morfologicamente subespecificados (veja-se NUNES, 2019); (iii) T finito em PB poder portar pessoa e número ou apenas número (vejam-se FERREIRA, 2000, 2009 e NUNES, 2008); e (iv) em tempos verbais sujeitos a empobrecimento, o traço de número de Té apagado (veja-se KATO; MARTINS; NUNES, a sair).

\section{REFERÊNCIAS}

BARBOSA, P.; DUARTE, M. E; KATO, M. A. A distribuição do sujeito nulo no português europeu e no português brasileiro. In: NUNES CORREIA, C.; GONÇALVES, A. (org.). Actas do XVI Encontro Nacional da Associação Portuguesa de Linguística. Lisboa: Associação Portuguesa de Linguística, 2001.p. 539-550.

BARBOSA, P.; DUARTE, M. E; KATO, M. Null subjects in European and Brazilian Portuguese. Journal of Portuguese Linguistics, v. 4, p. 11-52, 2005

BONET, E. Morphology after syntax: pronominal clitics in romance. 1991. Tese (Doutorado) - Massachusetts Institute of Technology, Cambridge, Mass., 1991.

CHAO, W. The interpretation of null subjects: Brazilian Portuguese. Cahiers Linguistiques d'Ottawa, v. 11, p. 69-74, 1983. 
CHOMSKY, N. Derivation by phase. In: KENTOWICZ, M. (org.). Ken hale: a life in language. Cambridge, Mass: MIT Press, 2001. p. 1-52.

DUARTE, M. E. Do pronome nulo ao pronome pleno: a trajetória do sujeito no português do Brasil. In: ROBERTS, I.; KATO, M. A. (org.). Português brasileiro: uma viagem diacrônica. Campinas: Editora da Unicamp. 1993. p. 107-128.

DUARTE, M. E. A perda do princípio "Evite Pronome" no português brasileiro. 1995. Tese (Doutorado) - Universidade Estadual de Campinas, Campinas, 1995.

DUARTE, M. E. The loss of the Avoid Pronoun principle in Brazilian Portuguese. In: KATO, M. A.; NEGRÃO, E. (org.). Brazilian Portuguese and the null subject parameter. Madrid \& Frankfurt am Main: Iberoamericana \& Vervuert, 2000. p. 17-36.

DUARTE, M. E. On the embedding of a syntactic change. Language variation in Europe: Papers from ICLaVEZ. Uppsala: Universitetstryckeriet, 2004.p. 145-155.

DUGUINE, M. Null arguments and linguistic variation. 2013. Tese (Doutorado) -Universidad del País Vasco/Université de Nantes, Vitoria-Gasteiz/Nantes, 2013.

FERREIRA, M. Argumentos nulos em português brasileiro. 2000. Dissertação (Mestrado) - Universidade Estadual de Campinas, Campinas, 2000.

FERREIRA, M. Null subjects and finite control in Brazilian Portuguese. In: NUNES, J. Nunes (org.). Minimalist essays on Brazilian Portuguese syntax. Amsterdam/Philadelphia: John Benjamins. 2009. p. 17-49.

FIGUEIREDO SILVA, M. C. 1996. A posição do sujeito no português brasileiro: frases finitas e infinitivas. Campinas: Editora da UNICAMP.

GALVES, C. A sintaxe do português brasileiro. Ensaios de Linguística, v. 13, p. 31-50, 1987.

GALVES, C. O enfraquecimento da concordância no português brasileiro. In: ROBERTS, I.; KATO, M. A. (org.). Português brasileiro: uma viagem diacrônica. Campinas: Editora da Unicamp. 1993. p. 387-408.

GALVES, C. Tópicos, sujeitos, pronomes e concordância no português brasileiro. Cadernos de Estudos Lingüísticos, v. 34, p. 7-21, 1998.

HOLMBERG, A.; NAYUDU, A. N; SHEEHAN, M. Three partial null-subject languages: a comparison of Brazilian Portuguese, Finnish and Marathi. Studia Linguistica v. 63, p. 59-97, 2009.

HORNSTEIN, N. Movement and control. Linguistic Inquiry, v. 30, p. 69-96, 1999.

HORNSTEIN, N. Move! A minimalist theory of construal. Oxford: Blackwell. 2001.

KATO, M. A. Strong pronouns, weak pronominals and the null subject parameter. Probus v. 11, p. 1-37, 1999.

KATO, M. A. The partial pro-drop nature and the restricted VS order in Brazilian Portuguese. In: KATO, M. A.; NEGRÃO, E. (org.). Brazilian Portuguese and the null subject parameter. Madrid \& Frankfurt am Main: Iberoamericana \& Vervuert. 2000. p. 223258.

KATO, M. A. Pronomes fortes e fracos na sintaxe do português brasileiro. Revista Portuguesa de Filologia, v. XXIV, p. 101-122, 2002.

Forum lingüístic., Florianópolis, v.17, número especial, p.4658-4672, jun. 2020. 
KATO, M. A.; MARTINS, A. M; NUNES, J. The syntax of Portuguese. Cambridge: Cambridge University Press. [a sair].

KATO, M. A.; NEGRÃO, E. (org.). Brazilian Portuguese and the null subject parameter. Madrid \& Frankfurt am Main: Iberoamericana \& Vervuert., 2000.

MARTINS, A. M; NUNES, J. Raising issues in Brazilian and European Portuguese. Journal of Portuguese Linguistics, v. 4, p. 53-77, 2005.

MARTINS, A. M; NUNES, J. Apparent hyper-raising in Brazilian Portuguese: agreement with topics across a finite CP. In: PANAGIOTIDIS, P. (org.). The complementiser phase: subjects and wh-dependencies. Oxford: Oxford University Press. 2010. p. 142-163.

MARTINS, A. M; NUNES, J. Brazilian and European Portuguese and Holmberg's 2005 typology of null subject languages. In: BAAUW, B., MERONI, L.; DRIJKONIGEN, F. (org.). Romance languages and linguistic theory, selected papers from 'Going Romance' 32. Amsterdam/Philadelphia: John Benjamins. [a sair].

MENON, O.; LOREGIAN-PENKAL, L. Variação no indivíduo e na comunidade: Tu/você no sul do Brasil. In: VANDRENSEN, P. (org.). Variação e mudança no português falado no Sul. Pelotas: Educat. 2002. p.147-188.

MERCHANT, J. The syntax of silence. Oxford: Oxford University Press. 2001.

MODESTO, M. On the identification of null arguments. 2000. Tese (Doutorado) -, University of Southern California, Los Angeles, 2000 .

MOREIRA DA SILVA, S. Études sur la symétrie et l'asymétrie sujet/objet dans le portugais du Brésil. 1983.Tese (Doutorado) Université de Paris VIII, 1983.

NEGRÃO, E. Anaphora in Brazilian Portuguese complement structures. 1986. Tese (Doutorado) - University of Wisconsin, Madisson, 1986.

NUNES, J. Inherent Case as a licensing condition for A-movement: the case of hyper-raising constructions in Brazilian Portuguese. Journal of Portuguese Linguistics, v. 7, p. 83-108, 2008.

NUNES, J. Subespecificação de traços-f e hiperalçamento em português brasileiro. In: FIGUEREDO, C.; ARAÚJO, E. (org.). Diálogos com Ribeiro: sobre gramática e história da língua portuguesa. Salvador: Edufba, 2015. p. 121-148.

NUNES, J. Subject and topic hyper-raising in Brazilian Portuguese: a case study on reference sets for economy computations. In: KATO, M. A.; ORDÓÑEZ, F. (org). The morphosyntax of Spanish and Portuguese in Latin America. Oxford: Oxford University Press, 2016. p. 107-134.

NUNES, J. Remarks on finite control and hyper-raising in Brazilian Portuguese. Journal of Portuguese Linguistics, v. 18, n.4, p. 1-50, 2017.

PAREDES SILVA, V. O retorno do pronome $t u$ à fala carioca. In: RONCARATI; ABRAÇADO, J. (org.). Português brasileiro: contato lingüístico, heterogeneidade, história. Rio de Janeiro: Sete Letras, 2003. p. 160-169.

PERLMUTTER, D. Deep and surface constraints in syntax. New York: Holt, Rinehart \& Winston. 1971. 
PETERSEN, M. C. O licenciamento do sujeito nulo em orações subjuntivas no português brasileiro: Contribuições para a Teoria de Controle por Movimento. 2011. Dissertação (Mestrado) - Universidade de São Paulo, São Paulo, 2011.

ROBERTS, I. A deletion analysis of null subjects. In: BIBERAUER, T. et al. (org.). Parametric variation. Cambridge: Cambridge University Press, 2010. p. 58-87.

RODRIGUES, C. Morphology and null subjects in Brazilian Portuguese. In: LIGHTFOOT, D. (org.). Syntactic effects of morphological change. Oxford: Oxford University Press. 2002. p. 160-178.

RODRIGUES, C. Impoverished morphology and A-movement out of case domains. 2004. Tese (Doutorado) - University of Maryland, College Park, 2004.

SAAB, A. On the notion of partial (non-)pro-drop in Romance. In: KATO, M. A.; ORDÓNEZ, F. (org). The morphosyntax of Spanish and Portuguese in Latin America. Oxford: Oxford University Press, 2016. p. 49-77.

URA, H. Varieties of raising and the feature-based bare phrase structure theory. MIT Working Papers in Linguistics, v. 7, 1994.

\section{(ㄷ) (1) $(9)$}

